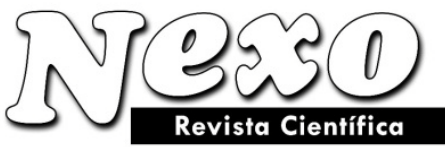

ISSN-E 1995-9516

Universidad Nacional de Ingeniería http://revistas.uni.edu.ni/index.php/Nexo http://dx.doi.org/10.5377/nexo.v28i2.3441

\title{
OPTIMIZACIÓN DE INDICADORES DE RENTABILIDAD MEDIANTE EL MODELO DE DEPRECIACIÓN ÁURICA EN NICARAGUA
}

\section{OPTIMIZATION OF PROFITABILITY INDICATORS THROUGH THE AURIC DEPRECIATION MODEL IN NICARAGUA}

\author{
Silva, F.M. ${ }^{1}$, Marenco, G.A. ${ }^{2}$ y Zelaya, C.E. ${ }^{3}$. \\ 1 Facultad de Ciencias Económicas y Sociales (FCES), división de estudios para graduados. Doctorado de \\ Ciencias Económicas. Universidad del Zulia (LUZ). \\ manfreddy22@hotmail.com \\ 2,3 Facultad de Ciencia, Tecnología y Ambiente (FCTyA), \\ Univers idad Centroamericana (UCA). Ingeniería Industria 1. \\ gerald.marenco@hotmail.com ; charlieze laya.cz@gmail.com
}

(recibido/received: 25-Mayo-2015; aceptado/accepted: 10-Diciembre-2015)

\section{RESUMEN}

La implementación de medidas fiscales tiende a disminuir el impacto de la carga tributaria sobre las actividades económicas empresariales. Por años se ha utilizado la depreciación como escudo fiscal, así que se planteó un nuevo modelo denominado Depreciación Áurica. Este planteamiento del modelo consistió en ponderar valores más altos en los primeros años de un activo con respecto a la sucesión de Fibonacci, para que sirviera a la mejora de los indicadores de rentabilidad: tasa interna de retorno (TIR) e índice de rentabilidad (IR). Se comparó este nuevo modelo con el de línea recta y depreciación acelerada (por suma de dígitos), a través del planteamiento de un escenario para la empresa con una anualidad mínima requerida para cumplir la premisa de rentabilidad; se disminuyó esta anualidad con la carga impositiva de1 30\% no sin antes aplicar tres métodos de depreciación. Al final, la TIR promedio y el IR corregido por impuestos, resultaron ser mayores con la aplicación del modelo áurico a tasas planificadas superiores al $16 \%$, pues a menores tasas no se permitió una rentabilidad apreciable por ningún método. Este modelo tiene, pues, la finalidad de servir como incentivo para la creación y formalización de nuevas empresas, le permite capitalización y supervivencia, y garantiza a la administración tributaria la recaudación de impuestos a largo plazo.

Palabras claves: Depreciación áurica; Tasa interna de retorno; Sucesión de Fibonacci; Índice de rentabilidad; Incentivo fiscal. 


\begin{abstract}
The implementation of fiscal measures tends to diminish the impact of the tax burden on business economic activities. For years has been used depreciation as a tax shield, so a new model called auric depreciation raised. This approach of the model consisted in we ighing higher values in the early years of an asset with regard to the Fibonacci sequence, so serve the improvement of profitability indicators: internal rate of return (IRR) and profitability index (IR). This new model with the straight line and accelerated depreciation (by sum of digits), was compared by the setting of a stage for the enterprise with a minimum annuity to carry the premise of profitability; this annuity with the $30 \%$ tax burden was reduced but not before applying three depreciation methods. At the end, average IRR and IR corrected for taxes, proved to be greater with the application of the Auric to planned growth rates higher than $16 \%$, as lower rates is not allowed an appreciable return by any method. This model has, therefore the purpose serve as an incentive for the creation and formalization of new companies, it allows capitalization and survival, and warrants to the tax administration revenue in the long run.
\end{abstract}

Keywords: Auric Depreciation; internal rate of return; Fibonacci sequence; profitability index; Tax incentive

\title{
INTRODUCCIÓN
}

El peso de los objetivos de supervivencia, rentabilidad y crecimiento de las organizaciones recae en las decisiones de inversión que se efectúan. Por lo tanto para tomar esta decisión es necesario conocer los flujos de dinero del proyecto, los cuales se determinan mediante la proyección de los estados financieros básicos a base de las estimaciones de mercado (Rosillo, 2005).

En esta proyección de los flujos de dinero se ve involucrado los impuestos recaudados por la administración tributaria de Nicaragua y una de las deducciones permitidas según la Ley 822, Ley de Concertación Tributaria (LCT) es la depreciación del activo fijo que genera renta. En Nicaragua el método más utilizado para depreciar un bien es el de línea recta, el cual no representa ningún incentivo para las nuevas empresas.

Los gobiernos, como se sabe, utilizan los impuestos para recaudar fondos públicos con tasas que persiguen un objetivo específicamente fiscal; pero el logro de este objetivo acarrea impactos negativos en los sectores productivos o sociales de la economía, por lo cual se procuran medidas que minimizan o esterilizan dichos impactos colaterales. Si ocurre un endeudamiento, es probable que baje la base imponible y se pagarían menos impuestos, y si la deuda es relativamente baja, su grado de impacto será menor, por lo que se perderá ventaja tributaria. Cuando se tiene mayor participación en la deuda, se consigue un mayor valor presente neto ajustado, con lo cual mejoraría la posición económica de la inversión (Gomero, 2014).

Los activos disminuyen su valor corregido a medida que envejecen, esto es, si se toma en un corte transversal la relación precio-edad de los bienes de equipo, es posible ajustar una función, generalmente no lineal, a partir de cuyas predicciones se puede estimar la depreciación económica como la tasa de variación del precio del activo con respecto a la edad, en un periodo determinado. Para este efecto, la depreciación recoge el conjunto de las minoraciones, en valor, cantidad o calidad, del acervo productivo de una empresa o, incluso a nivel agregado, de una economía nacional, durante un determinado periodo de tiempo. Estas minoraciones pueden manifestarse tanto en forma de abandonos completos y definitivos de equipos productivos, como en la pérdida parcial de los mismos o de su capacidad de producir bienes económicos (Ruiz, 1992). 
Las causas de la depreciación se pueden agrupar en dos categorías fundamentales: el deterioro (característica inherente a los bienes de equipo, vinculada al envejecimiento y desgaste de los equipos) y la obsolescencia (característica que sobreviene como consecuencia de circunstancias ajenas a los propios equipos productivos que la experimentan). Asumir que la depreciación pueda estar causada por deterioro y obsolescencia conlleva, inevitablemente, a la aceptación de una tasa de depreciación variable. Una búsqueda por esta tasa en trabajos literarios de los años setenta llevó a querer adjudicar al volumen de depreciación, y a la tasa de depreciación en particular, la condición de variable de decisión empresarial. Estos trabajos fueron los de Feldstein y Foot (1971) y Eisner (1972), quienes considerando la variable depreciación como endógena, intentaron modelizar las decisiones empresariales. Nickell (1975), en cambio, plantea un modelo de generaciones con explicitación únicamente del deterioro económico y obtiene una regla de retiros en donde el periodo medio de vida figura como variable endógena. Otros trabajos, como los de Mizon (1974), Malcomson (1975) y Malcomson y Prior (1979), relacionan el progreso técnico incorporado en la nueva inversión y analizan la duración óptima del equipo productivo (Ruiz, 1992).

Fibonacci hacia el año 1202 descubrió una simple serie numérica que creaba las proporciones naturales de los objetos en el universo. Es así como nace la sucesión de números: 0, 1, 1, 2, 3, 5, 8, 13, etc. Esta sucesión está formada por la suma de los dos números inmediatos anteriores, empezando por cero y uno. La ventaja de esta serie es que, a medida que avanza, los siguientes valores se pueden obtener a través de un factor denominado número áureo. Actualmente, los softwares actualizados pueden generar gran cantidad de información a partir de esta serie. Los operadores utilizan los niveles de retracción de Fibonacci (factor áureo de decrecimiento) como niveles de soporte y resistencia, que cumplen las expectativas del mercado bursátil; es decir, se utiliza en las operaciones financieras para calcular los niveles de compra y venta para obtener ganancias, sin embargo solamente se utilizan de cuatro a seis factores para corregir momentos determinados del valor de bolsa (Garza, 2014).

El objetivo general del presente artículo es plantear matemáticamente un modelo de depreciación que considere la sucesión de Fibonacci como parámetro que establezca las cuotas de depreciación anuales, mediante la consideración de los años de vida útil para activos fijos en la LCT, a fin de que se considere la rentabilidad a través del aprovechamiento de los escudos fiscales y el incentivo para las inversiones. Como objetivos específicos se plantean: evaluar financieramente el modelo de depreciación áurica, verificar la incidencia de los costos de depreciación en los flujos de efectivo y comparar estos resultados con los actuales métodos de depreciación que se utilizan en el país.

La formulación de un modelo de depreciación áurica se justifica con la pretensión de corregir el valor contable del deterioro y la obsolescencia de los activos fijos mediante una secuencia a porcentajes no lineales que explicarían los efectos en un periodo determinado como una ponderación sumada de los efectos sucesivos con base en la sucesión de Fibonacci. Esto es, que otorga valores más altos de depreciación para los primeros años con el fin de lograr una mejor capitalización inicial, lo cual estaría dirigido a las nuevas empresas que invierten en activos para el rendimiento de efectivo, mediante una disminución en la base imponible, pero que, una vez recuperada la mayor parte de la inversión por recuperación, se puedan afrontar los montos impositivos con mayor disponibilidad de efectivo.

\section{MATERIALES Y MÉTODOS}

La optimización de los indicadores de rentabilidad a partir del modelo de depreciación áurica parte de las definiciones propias de los métodos para la evaluación de inversiones. Las premisas del modelo se establecieron sobre la marcha del planteamiento, según convino.

Según Baca (2007), una variable importante en la toma de decisiones económicas es el valor presente neto (VPN). El VPN puede calcularse como la sumatoria de los n-ésimos flujos netos de efectivo (FNE) con su 
valor trasladado a presente a una tasa mínima atractiva de rendimiento (TMAR) según el período de tiempo donde se encuentre $(\mathrm{k})$, más el valor de salvamento (VS) trasladado a presente en un período específico $(\mathrm{t})$, menos el valor de la inversión $(\mathrm{P})$ :

$\mathrm{VPN}=-\mathrm{P}+\sum_{\mathrm{k}=1}^{\mathrm{n}} \frac{\mathrm{FNE}_{\mathrm{k}}}{(1+\mathrm{TMAR})^{\mathrm{k}}}+\frac{\mathrm{VS}}{(1+\mathrm{TMAR})^{\mathrm{t}}}$

Para la construcción del presente modelo, se tomó la premisa que el valor del flujo neto de efectivo para los períodos es equivalente a anualidades iguales (A). Esto se hace con la finalidad de facilitar los cálculos y evitar abordar la casuística de flujos que generaría múltiples probabilidades:

$\mathrm{A}\left[\frac{\left(1+\mathrm{TMAR}^{\mathrm{n}}-1\right.}{\operatorname{TMAR}\left(1+\mathrm{TMAR}^{\mathrm{n}}\right.}\right]=\sum_{\mathrm{k}=1}^{\mathrm{n}} \frac{\mathrm{FNE}_{\mathrm{k}}}{\left(1+\mathrm{TMAR}^{\mathrm{k}}\right.}$

No se consideró tampoco el valor de salvamento, porque su monto se presta también a una gama de posibilidades y su tratamiento fiscal es diferente (ganancias y pérdidas de capital). Por tanto, eliminando el valor de salvamento y sustituyendo (2) en (1), el modelo se simplifica como:

$V P N=-P+A\left[\frac{(1+\text { TMAR })^{n}-1}{\operatorname{TMAR}\left(1+\text { TMAR }^{n}\right.}\right]$

Otra variable relevante para la toma de decisiones económicas es el índice de rentabilidad (IR), el cual se calcula como el cociente del valor presente neto (VPN) y el valor absoluto de la inversión (P):

$\mathrm{IR}=\frac{\mathrm{VPN}}{|\mathrm{P}|}$

El punto decisivo del IR es su comparación con la TMAR mediante dos casos: si IR es mayor o igual que TMAR, se acepta la inversión; en caso contrario, se rechaza. La construcción del modelo empezó ahora por declarar la igua ldad entre IR y TMAR como condición mínima para poder realizar la inversión:

$\mathrm{IR}=\mathrm{TMAR}$

Despejando VPN en la ecuación (4):

$\mathrm{VPN}=\mathrm{TMAR} * \mathrm{P}$

Sustituyendo (6) en (3):

$\operatorname{TMAR} * \mathrm{P}=-\mathrm{P}+\mathrm{A}\left[\frac{(1+\mathrm{TMAR})^{\mathrm{n}}-1}{\operatorname{TMAR}\left(1+\mathrm{TMAR}^{\mathrm{n}}\right.}\right]$

Despejando el valor de las anualidades:

$$
A=P\left[\frac{\operatorname{TMAR}\left(1+\text { TMAR }^{\mathrm{n}+1}\right.}{(1+\mathrm{TMAR})^{\mathrm{n}}-1}\right]
$$


La interpretación de esta ecuación explica que se puede calcular la anualidad mínima requerida de parte del activo en el que se invirtió para obtener un índice de rentabilidad (llamado IR planificado sin impuestos) equivalente a la tasa mínima atractiva de rendimiento (TMAR planificada sin aplicar impuestos) y, con esta condic ión mínima, aceptar invertir o no. Bajo estas condiciones es posible construir una tabla de flujos de efectivo (Tabla 1). Esta construcción de flujo de efectivo no considera los gastos por depreciación ni los impuestos. Para contemplar las depreciaciones, se considera la variante años de vida útil de los activos fijos (Tabla 2), la cual se encuentra contemplada en LCT y se estratifican dichos activos en 8 rubros de acuerdo a su duración.

Tabla 1. Construcción de flujos de efectivo a partir de anualidad mínima requerida

\begin{tabular}{|c|c|c|c|c|c|c|c|}
\hline Períodos & 0 & 1 & 2 & 3 & $\ldots$ & $\mathrm{n}-1$ & $\mathrm{~N}$ \\
\hline Flujos netos de efectivo & $-\mathrm{P}$ & $\mathrm{A}$ & $\mathrm{A}$ & $\mathrm{A}$ & $\ldots$ & $\mathrm{A}$ & $\mathrm{A}$ \\
\hline
\end{tabular}

Tabla 2. Clasificación de activos según el período de vida útil

\begin{tabular}{|c|c|}
\hline Período de vida útil & Activos \\
\hline 2 años & $\begin{array}{l}\text { Equipos de computación. } \\
\text { Equipos para medios de comunicación. }\end{array}$ \\
\hline 3 años & Vehículos de empresa de alquiler. \\
\hline 5 años & $\begin{array}{l}\text { Vehículos de uso particular usados en rentas de actividades económicas. } \\
\text { Maquinarias y equipos distintas a fija en bien inmóvil o a no adherida a la } \\
\text { planta. } \\
\text { Equipo de empresas agroindustriales. } \\
\text { Maquinaria y equipos agrícolas. } \\
\text { Mobiliar ios y equipo de oficina. } \\
\text { Equipos de comunicación. }\end{array}$ \\
\hline 7 años & Equipo industrial no adherido permanentemente a la planta. \\
\hline 8 años & $\begin{array}{l}\text { Equipos de transporte distinto de colectivo, carga, de empresa de alquiler o } \\
\text { de uso en actividades económicas }\end{array}$ \\
\hline 10 años & $\begin{array}{l}\text { Edificios indus triales. } \\
\text { Residencia del propietario cuando esté ubicado en finca destinada a } \\
\text { explotación agropecuaria. } \\
\text { Instalaciones fijas en explotaciones agropecuarias. } \\
\text { Maquinaria y equipos industriales fijos en un bien inmóvil. } \\
\text { Ascensores, elevadores y unidades centrales de aire acondicionado. }\end{array}$ \\
\hline 20 años & Edificios comerciales \\
\hline 30 años & Edificios de alqu iler \\
\hline
\end{tabular}

Conocidos los modelos de depreciación por línea recta y la depreciación acelerada (se tomó el método de suma de dígitos porque es aplicable a la mayoría de activos), se plantea el método áurico, el cual empieza por la consideración de que un modelo de la sucesión de Fibonacci necesariamente utiliza la proporción áurica que a valores mayores se aproxima a $(1+\sqrt{5}) / 2 \approx$ 1,61803, para calcular los porcentajes de depreciación en cada plazo de los activos fijos. Estos tienen al inicio de su vida útil un valor contable que es necesario ir recuperando, por lo tanto se procede a ponderar la serie de Fibonacci (tomada desde 1 y no de cero), empezando por su valor más alto (o término enésimo) en relación al número de años de vida útil del activo según el Reglamento de la LCT. Por ejemplo, se considera que para un período de dos años hay dos números de la serie: 1 y 1; a cada período le corresponde un valor de depreciación de $50 \%$ y $50 \%$. En 
cambio, para un activo de cinco años, la serie toma los valores de $1,1,2,3$ y 5 . Su primer valor será de $5 /(5+3+2+1+1)=5 / 12$, el segundo valor será de $3 / 12$ y así hasta el quinto período.

Para lograr un panorama más delimitado, se consideró una base imponible del 30\% sobre la utilidad antes de impuestos (UAI, esto es la utilidad bruta restando el valor de la depreciación). Una vez obtenida la utilidad después de impuestos (UDI), se construyen los flujos de efectivo sumándole a esta UDI el valor de la depreciación (Tabla 3).

Tabla 3. Flujos de efectivo corregidos luego de impuestos

\begin{tabular}{|c|c|c|c|c|c|c|c|}
\hline Períodos & 0 & 1 & 2 & 3 & $\ldots$ & n-1 & $\mathrm{N}$ \\
\hline Beneficio anual & $-\mathrm{P}$ & A & A & A & $\ldots$ & A & A \\
\hline De pre ciación & & $D_{1}$ & $\mathrm{D}_{2}$ & $D_{3}$ & ... & $D_{n-1}$ & $D_{n}$ \\
\hline UAI & & A- $D_{1}$ & $\mathrm{~A}-\mathrm{D}_{2}$ & $\mathrm{~A}-\mathrm{D}_{3}$ & $\ldots$ & $A-D_{n-1}$ & A- $D_{n}$ \\
\hline Impuestos & & $30 \% * \mathrm{UAI}_{1}$ & $30 \% * \mathrm{UAI}_{2}$ & $30 \% * \mathrm{UAI}_{3}$ & $\ldots$ & $30 \% * \mathrm{UAI}_{\mathrm{n}-1}$ & $30 \% * \mathrm{UAI}_{\mathrm{n}}$ \\
\hline UDI & & $70 \% * \mathrm{UAI}_{1}$ & $70 \% * \mathrm{UAI}_{2}$ & $70 \% * \mathrm{UAI}_{3}$ & $\ldots$ & $70 \% * \mathrm{UAI}_{\mathrm{n}-1}$ & $70 \% * \mathrm{UAI}_{\mathrm{n}}$ \\
\hline Depreciación & & $D_{1}$ & $D_{2}$ & $D_{3}$ & ... & $D_{n-1}$ & $D_{n}$ \\
\hline FNE & $-\mathrm{P}$ & $\mathrm{UDI}_{1+} \boldsymbol{D}_{1}$ & $\mathrm{UDI}_{2}+\boldsymbol{D}_{2}$ & $\mathrm{UDI}_{3}+\boldsymbol{D}_{3}$ & $\ldots$ & $\mathrm{UDI}_{\mathrm{n}-1}+\boldsymbol{D}_{\boldsymbol{n}-1}$ & $\mathrm{UDI}_{\mathrm{n}}+\boldsymbol{D}_{\boldsymbol{n}}$ \\
\hline
\end{tabular}

Lo anterior indica que el IR planificado al inicio (que igualado a la tasa mínima atractiva de rendimiento se podía obtener anualidades iguales), sufre cambios por el pago de los impuestos. Este nuevo índice de rentabilidad, que se presume menor por el monto impositivo, se conoce como índice de rentabilidad corregido (IR corregido). Si la TMAR planificada fue razonablemente mayor, el proyecto es rentable por el criterio de que VPN es mayor que cero. Para analizar este efecto, también se calculó la tasa interna de retorno (TIR) a fin de evaluar si la inversión inicial no se ve significativamente afectada por la tasa impositiva al final del período de flujos de efectivo.

Con los parámetros anteriores, se formularon los flujos de efectivo y se desarrollaron cálculos en el software Excel para los tres modelos estudiados por cada uno de los ocho tipos de activo y se evaluaron los criterios para toma de decisiones en inversiones tomando los porcentajes uno a uno para la TMAR planificada desde $1 \%$ a $100 \%$, es decir que se obtuvieron datos para 2400 casos de flujo de efectivo. Sin embargo, el anális is uno a uno de todos los casos de activos es una técnica laboriosa, por lo que se optó por obtener una tasa interna de rendimiento promedio (TIR promedio) $\mathrm{y}$ un índice de rentabilidad corregido promedio (IR corregido promedio) de estos ocho tipos de activo en atención a su duración, a fin de simplificar el análisis de los datos a observaciones más puntuales.

La información referida a la formalización de empresas en Managua, para evaluar la ventaja de un incentivo fiscal por depreciación fue obtenida vía correo electrónico con la Ventanilla Única de Inversiones (VUI).

\section{RESULTADOS}

Se obtuvo el modelo completo de depreciación áurica (Tabla 4) a partir de los años de vida útil de los activos fijos, según el Reglamento de la LCT.

Una vez aplicado los tres modelos de depreciación (por línea recta, depreciación acelerada y áurica), se obtuvo la construcción de flujos de efectivo con las premisas planteadas. La inversión es igual a la unidad, para estudiar el caso general (Tabla 5). 
Silva, F.M., Marenco, G.A. y Zelaya, C.E.

Tabla 4. Construcción del modelo de depreciación áurica

Porcentajes de activo (\%)

\begin{tabular}{|c|c|c|c|c|c|c|c|c|}
\hline Año & 2 & 3 & 5 & 7 & 8 & 10 & 20 & 30 \\
\hline 1 & 50,0000 & 50,0000 & 41,6667 & 39,3939 & 38,8889 & 38,4615 & 38,1988 & 38,1966 \\
\hline 2 & 50,0000 & 25,0000 & 25,0000 & 24,2424 & 24,0741 & 23,7762 & 23,6081 & 23,6068 \\
\hline 3 & & 25,0000 & 16,6667 & 15,1515 & 14,8148 & 14,6853 & 14,5906 & 14,5898 \\
\hline 4 & & & 8,3333 & 9,0909 & 9,2593 & 9,0909 & 9,0175 & 9,0170 \\
\hline 5 & & & 8,3333 & 6,0606 & 5,5556 & 5,5944 & 5,5731 & 5,5728 \\
\hline 6 & & & & 3,0303 & 3,7037 & 3,4965 & 3,4444 & 3,4442 \\
\hline 7 & & & & 3,0303 & 1,8519 & 2,0979 & 2,1287 & 2,1286 \\
\hline 8 & & & & & 1,8519 & 1,3986 & 1,3156 & 1,3156 \\
\hline 9 & & & & & & 0,6993 & 0,8131 & 0,8131 \\
\hline 10 & & & & & & 0,6993 & 0,5025 & 0,5025 \\
\hline 11 & & & & & & & 0,3106 & 0,3106 \\
\hline 12 & & & & & & & 0,1920 & 0,1919 \\
\hline 13 & & & & & & & 0,1186 & 0,1186 \\
\hline 14 & & & & & & & 0,0734 & 0,0733 \\
\hline 15 & & & & & & & 0,0452 & 0,0453 \\
\hline 16 & & & & & & & 0,0282 & 0,0280 \\
\hline 17 & & & & & & & 0,0169 & 0,0173 \\
\hline 18 & & & & & & & 0,0113 & 0,0107 \\
\hline 19 & & & & & & & 0,0056 & 0,0066 \\
\hline 20 & & & & & & & 0,0056 & 0,0041 \\
\hline 21 & & & & & & & & 0,0025 \\
\hline 22 & & & & & & & & 0,0016 \\
\hline 23 & & & & & & & & 0,0010 \\
\hline 24 & & & & & & & & 0,0006 \\
\hline 25 & & & & & & & & 0,0004 \\
\hline 26 & & & & & & & & 0,0002 \\
\hline 27 & & & & & & & & 0,0001 \\
\hline 28 & & & & & & & & 0,0001 \\
\hline 29 & & & & & & & & 0,0000 \\
\hline 30 & & & & & & & & 0,0000 \\
\hline
\end{tabular}

Nexo Revista Científica Vol. 28, No. 02, pp. 67-82/Diciembre 2015 
Silva, F.M., Marenco, G.A. y Zelaya, C.E.

Tabla 5. Modelo de flujos de efectivo para los tres métodos de depreciación estudiados

Ejemplo: TMAR planificada de $25 \%=$ IR planificado de 25\%. Caso: Activo de 5 años

\begin{tabular}{|c|c|c|c|c|c|c|}
\hline \multicolumn{7}{|c|}{$\begin{array}{ll}\text { Períodos } & 0\end{array}$} \\
\hline \multicolumn{7}{|c|}{ Depreciación por líne a recta } \\
\hline Ingreso anual & & 0,4648 & 0,4648 & $\mathrm{C} \$ 0,4648$ & $\mathrm{C} \$ \quad 0,4648$ & C\$ 0,4648 \\
\hline Depreciación & & 0,2000 & C\$ $\quad 0,2000$ & C\$ 0,2000 & C\$ 0,2000 & 0,2000 \\
\hline UAI & & 0,2648 & $C \$ \quad 0,2648$ & C\$ 0,2648 & C\$ $\quad 0,2648$ & C\$ $\quad 0,2648$ \\
\hline Impuesto & & 0,0794 & C\$ $\quad 0,0794$ & C\$ 0,0794 & C\$ $\quad 0,0794$ & C\$ $\quad 0,0794$ \\
\hline UDI & & 0,1854 & C\$ $\quad 0,1854$ & C\$ 0,1854 & C\$ $\quad 0,1854$ & C\$ 0,1854 \\
\hline Depreciación & & 0,2000 & C\$ $\quad 0,2000$ & C\$ 0,2000 & C $\$ \quad 0,2000$ & C\$ 0,2000 \\
\hline FNE & C\$ $(1,0000)$ & $\mathbf{0 , 3 8 5 4}$ & C\$ $\quad \mathbf{0 , 3 8 5 4}$ & C\$ 0,3854 & C\$ $\mathbf{0 , 3 8 5 4}$ & C\$ $\quad 0,3854$ \\
\hline \multicolumn{7}{|c|}{ TIR $=26,7629 \%$} \\
\hline \multicolumn{7}{|c|}{ IR corre gido $=3,6357 \%$} \\
\hline \multicolumn{7}{|c|}{$\mathrm{VPN}=\mathrm{C} \$ \mathbf{0 , 0 3 6 4}$} \\
\hline \multicolumn{7}{|c|}{ De pre ciación acele rada } \\
\hline Ingreso anual & & $\mathrm{C} \$ 0,4648$ & $\mathrm{C} \$ 0,4648$ & $\mathrm{C} \$ 0,4648$ & $\mathrm{C} \$ 0,4648$ & $\mathrm{C} \$ 0,4648$ \\
\hline Depreciación & & C\$0,3333 & C\$0,2667 & C\$0,2000 & C\$0,1333 & C\$0,0667 \\
\hline UAI & & $\mathrm{C} \$ 0,1315$ & $\mathrm{C} \$ 0,1981$ & $\mathrm{C} \$ 0,2648$ & $\mathrm{C} \$ 0,3315$ & $\mathrm{C} \$ 0,3981$ \\
\hline Impuesto & & $\mathrm{C} \$ 0,0394$ & $\mathrm{C} \$ 0,0594$ & $\mathrm{C} \$ 0,0794$ & $\mathrm{C} \$ 0,0994$ & $\mathrm{C} \$ 0,1194$ \\
\hline UDI & & $\mathrm{C} \$ 0,0920$ & $\mathrm{C} \$ 0,1387$ & $\mathrm{C} \$ 0,1854$ & $\mathrm{C} \$ 0,2320$ & $\mathrm{C} \$ 0,2787$ \\
\hline Depreciación & & $\mathrm{C} \$ 0,3333$ & $C \$ 0,2667$ & $C \$ 0,2000$ & $C \$ 0,1333$ & $C \$ 0,0667$ \\
\hline FNE & $\operatorname{C}(\mathbf{1}, 0000)$ & C\$0,4254 & C\$0,4054 & C\$0,3854 & C\$0,3654 & C\$0,3454 \\
\hline \multicolumn{7}{|c|}{ TIR $=28,0060 \%$} \\
\hline \multicolumn{7}{|c|}{ IR corre gido $=\mathbf{5 , 9 8 5 8} \%$} \\
\hline \multicolumn{7}{|c|}{$\mathrm{VPN}=\mathrm{C} \$ 0,0599$} \\
\hline \multicolumn{7}{|c|}{ De preciación áurica } \\
\hline Ingreso anual & & $\mathrm{C} \$ 0,4648$ & $\mathrm{C} \$ 0,4648$ & $\mathrm{C} \$ 0,4648$ & $\mathrm{C} \$ 0,4648$ & $\mathrm{C} \$ 0,4648$ \\
\hline Depreciación & & C\$0,4167 & C\$0,2500 & C\$0,1667 & $\mathrm{C} \$ 0,0833$ & C\$0,0833 \\
\hline UAI & & $\mathrm{C} \$ 0,0481$ & $\mathrm{C} \$ 0,2148$ & $\mathrm{C} \$ 0,2981$ & $\mathrm{C} \$ 0,3815$ & $\mathrm{C} \$ 0,3815$ \\
\hline Impuesto & & $\mathrm{C} \$ 0,0144$ & $\mathrm{C} \$ 0,0644$ & $\mathrm{C} \$ 0,0894$ & $\mathrm{C} \$ 0,1144$ & $\mathrm{C} \$ 0,1144$ \\
\hline UDI & & $\mathrm{C} \$ 0,0337$ & $\mathrm{C} \$ 0,1504$ & $\mathrm{C} \$ 0,2087$ & $\mathrm{C} \$ 0,2670$ & $\mathrm{C} \$ 0,2670$ \\
\hline Depreciación & & $\mathrm{C} \$ 0,4167$ & $\mathrm{C} \$ 0,2500$ & $\mathrm{C} \$ 0,1667$ & $\mathrm{C} \$ 0,0833$ & $\mathrm{C} \$ 0,0833$ \\
\hline FNE & $C \$(1,0000)$ & C\$0,4504 & C\$0,4004 & C\$0,3754 & C\$0,3504 & C\$0,3504 \\
\hline \multicolumn{7}{|c|}{ TIR $=28,4114 \%$} \\
\hline \multicolumn{7}{|c|}{ IR corre gido $=6,7032 \%$} \\
\hline \multicolumn{7}{|c|}{$\mathrm{VPN}=\mathrm{C} \$ \mathbf{0 , 0 6 7 0}$} \\
\hline
\end{tabular}

Una vez obtenidos los 2400 casos para TIR y el mismo número para IR corregido, y promediados por cada unidad porcentual, se obtuvieron las gráficas respectivas para estos indicadores (TIR promedio, figura 1; IR corregido promedio, figura 2).

Nexo Revista Científica Vol. 28, No. 02, pp. 67-82/Diciembre 2015 


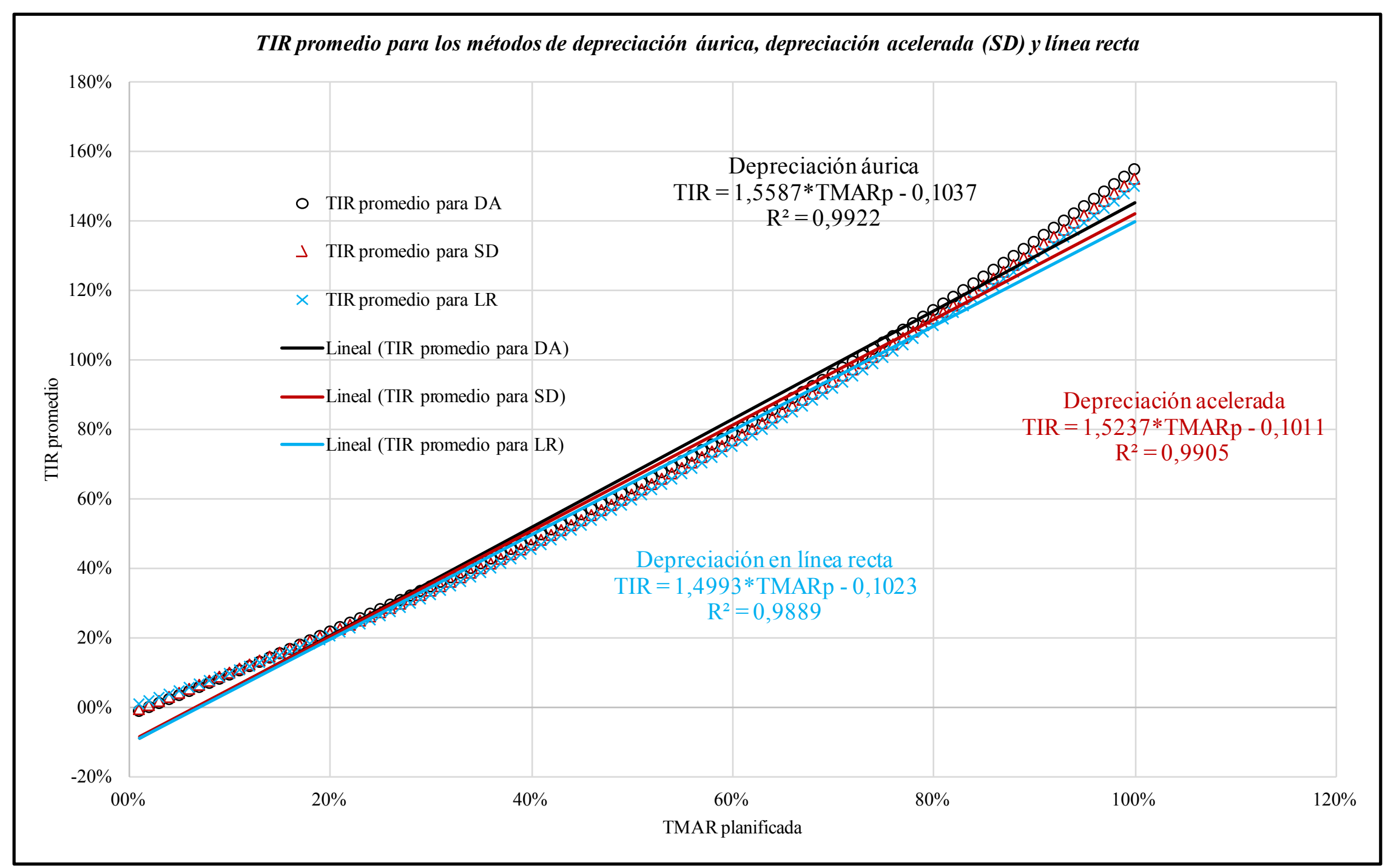

Fig. 1. TIR promedio para los métodos de depreciación áurica, depreciación acelerada y línea recta

Nexo Revista Científica Vol. 28, No. 02,pp. 67-82/Diciembre 2015 


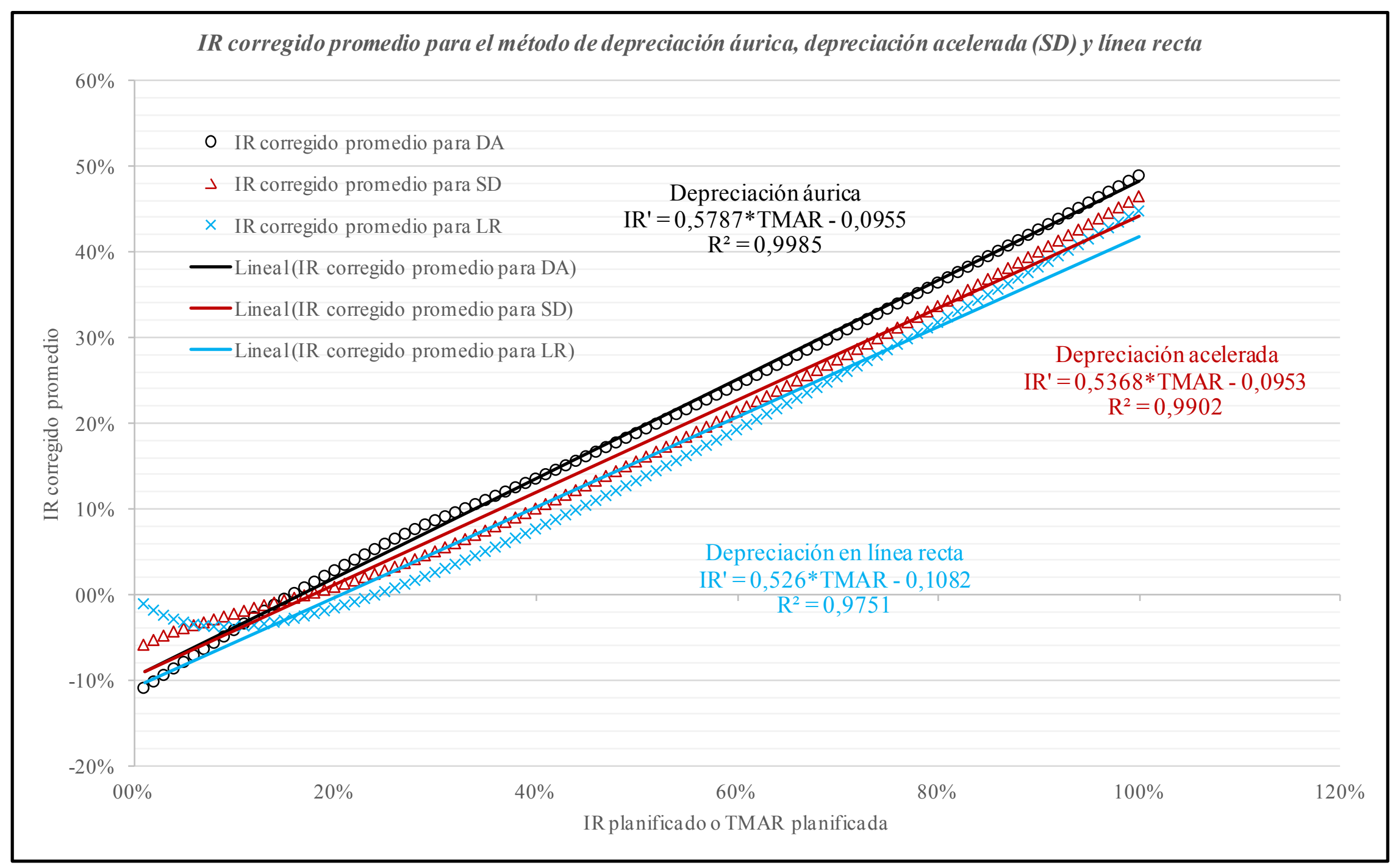

Fig. 2. IR corregido promedio para el método de depreciación áurica, depreciación acelerada y línea recta 
Silva, F.M., Marenco, G.A. y Zelaya, C.E.

Tabla 6. Anális is de puntos para TIR promedio

\begin{tabular}{|c|c|c|c|c|}
\hline $\begin{array}{c}\text { TMAR } \\
\text { planificada }\end{array}$ & $\begin{array}{l}\text { TIR prome dio para } \\
\text { depreciación áurica }\end{array}$ & $\begin{array}{l}\text { TIR prome dio para } \\
\text { depreciación ace le rada }\end{array}$ & $\begin{array}{l}\text { TIR prome dio para } \\
\text { líne a recta }\end{array}$ & Mejor método \\
\hline $1,00 \%$ & $-1,11 \%$ & $-0,59 \%$ & $0,91 \%$ & LR \\
\hline $2,00 \%$ & $0,02 \%$ & $0,58 \%$ & $1,83 \%$ & LR \\
\hline $3,00 \%$ & $1,16 \%$ & $1,74 \%$ & $2,77 \%$ & $\overline{\mathrm{LR}}$ \\
\hline $4,00 \%$ & $2,31 \%$ & $2,91 \%$ & $3,72 \%$ & LR \\
\hline $5,00 \%$ & $3,47 \%$ & $4,07 \%$ & $4,69 \%$ & $\overline{\mathrm{LR}}$ \\
\hline $6,00 \%$ & $4,63 \%$ & $5,24 \%$ & $5,67 \%$ & LR \\
\hline $7,00 \%$ & $5,80 \%$ & $6,40 \%$ & $6,65 \%$ & LR \\
\hline $8,00 \%$ & $6,97 \%$ & $7,57 \%$ & $7,65 \%$ & $\overline{\mathrm{LR}}$ \\
\hline $9,00 \%$ & $8,16 \%$ & $8,74 \%$ & $8,66 \%$ & SD \\
\hline $10,00 \%$ & $9,36 \%$ & $9,90 \%$ & $9,68 \%$ & SD \\
\hline $11,00 \%$ & $10,58 \%$ & $11,07 \%$ & $10,72 \%$ & SD \\
\hline $12,00 \%$ & $11,80 \%$ & $12,25 \%$ & $11,76 \%$ & SD \\
\hline $13,00 \%$ & $13,03 \%$ & $13,39 \%$ & $12,81 \%$ & SD \\
\hline $14,00 \%$ & $14,26 \%$ & $14,54 \%$ & $13,87 \%$ & SD \\
\hline $15,00 \%$ & $15,50 \%$ & $15,67 \%$ & $14,95 \%$ & SD \\
\hline $16,00 \%$ & $16,73 \%$ & $16,79 \%$ & $16,03 \%$ & SD \\
\hline $17,00 \%$ & $17,96 \%$ & $17,92 \%$ & $17,13 \%$ & $\overline{\mathrm{DA}}$ \\
\hline $18,00 \%$ & $19,21 \%$ & $19,06 \%$ & $18,23 \%$ & $\overline{\mathrm{DA}}$ \\
\hline $19,00 \%$ & $20,48 \%$ & $20,20 \%$ & $19,35 \%$ & $\overline{\mathrm{DA}}$ \\
\hline $20,00 \%$ & $21,75 \%$ & $21,36 \%$ & $20,48 \%$ & $\overline{\mathrm{DA}}$ \\
\hline $21,00 \%$ & $23,03 \%$ & $22,53 \%$ & $21,61 \%$ & $\overline{\mathrm{DA}}$ \\
\hline$\ldots$ & $\ldots$ & $\ldots$ & $\ldots$ & $\ldots$ \\
\hline $100,00 \%$ & $154,83 \%$ & $152,08 \%$ & $149,90 \%$ & $\overline{\mathrm{DA}}$ \\
\hline
\end{tabular}

Nexo Revista Científica Vol. 28, No. 02, pp. 67-82/Diciembre 2015 
Tabla 7. Anális is de puntos para IR corregido

\begin{tabular}{|c|c|c|c|c|}
\hline $\begin{array}{c}\text { Índice de Rentabilidad } \\
\text { planificado }\end{array}$ & $\begin{array}{l}\text { IR corre gido promedio } \\
\text { para depreciación áurica }\end{array}$ & $\begin{array}{l}\text { IR corre gido prome dio } \\
\text { para de preciación } \\
\text { ace le rada }\end{array}$ & $\begin{array}{l}\text { IR corre gido promedio } \\
\text { para líne a recta }\end{array}$ & Mejor método \\
\hline $1,00 \%$ & $-10,75 \%$ & $-5,72 \%$ & $-0,94 \%$ & LR \\
\hline $2,00 \%$ & $-10,00 \%$ & $-5,16 \%$ & $-1,68 \%$ & LR \\
\hline $3,00 \%$ & $-9,24 \%$ & $-4,65 \%$ & $-2,28 \%$ & $\overline{\mathrm{LR}}$ \\
\hline $4,00 \%$ & $-8,48 \%$ & $-4,17 \%$ & $-2,73 \%$ & LR \\
\hline $5,00 \%$ & $-7,72 \%$ & $-3,79 \%$ & $-3,08 \%$ & LR \\
\hline $6,00 \%$ & $-6,95 \%$ & $-3,44 \%$ & $-3,33 \%$ & $\overline{\mathrm{LR}}$ \\
\hline $7,00 \%$ & $-6,22 \%$ & $-3,11 \%$ & $-3,50 \%$ & SD \\
\hline $8,00 \%$ & $-5,49 \%$ & $-2,76 \%$ & $-3,59 \%$ & SD \\
\hline $9,00 \%$ & $-4,75 \%$ & $-2,42 \%$ & $-3,63 \%$ & SD \\
\hline $10,00 \%$ & $-4,00 \%$ & $-2,10 \%$ & $-3,61 \%$ & SD \\
\hline $11,00 \%$ & $-3,23 \%$ & $-1,76 \%$ & $-3,54 \%$ & SD \\
\hline $12,00 \%$ & $-2,47 \%$ & $-1,40 \%$ & $-3,43 \%$ & SD \\
\hline $13,00 \%$ & $-1,75 \%$ & $-1,10 \%$ & $-3,28 \%$ & SD \\
\hline $14,00 \%$ & $-1,04 \%$ & $-0,80 \%$ & $-3,09 \%$ & SD \\
\hline $15,00 \%$ & $-0,35 \%$ & $-0,51 \%$ & $-2,88 \%$ & $\overline{\mathrm{DA}}$ \\
\hline $16,00 \%$ & $0,32 \%$ & $-0,24 \%$ & $-2,63 \%$ & $\overline{\mathrm{DA}}$ \\
\hline $17,00 \%$ & $0,98 \%$ & $0,05 \%$ & $-2,36 \%$ & $\overline{\mathrm{DA}}$ \\
\hline $18,00 \%$ & $1,65 \%$ & $0,36 \%$ & $-2,07 \%$ & $\overline{\mathrm{DA}}$ \\
\hline $19,00 \%$ & $2,33 \%$ & $0,69 \%$ & $-1,76 \%$ & $\overline{\mathrm{DA}}$ \\
\hline$\ldots$ & $\ldots$ & $\ldots$ & $\ldots$ & $\ldots$ \\
\hline $100,00 \%$ & $49,06 \%$ & $46,62 \%$ & $44,91 \%$ & $\overline{\mathrm{DA}}$ \\
\hline
\end{tabular}


Con respecto a la formación de nuevas empresas, se proporcionan los datos emitidos por la Ventanilla Única de Inversiones en el departamento de Managua (Tabla 6), la cual muestra un descenso de registro en el último año con una proyección de menor número de empresas para finales del presente año. Estos índices de la capital pueden ser significativos para el registro de empresas a nivel nacional.

Tabla 8. Información sobre empresas registradas en la Ventanilla Única de Inversiones de Managua

\begin{tabular}{|c|c|c|}
\hline Año & Número de empres as registradas & Crecimiento \\
\hline $\mathbf{2 0 1 0}$ & 571 & - \\
\hline $\mathbf{2 0 1 1}$ & 643 & $12,61 \%$ \\
\hline $\mathbf{2 0 1 2}$ & 772 & $20,06 \%$ \\
\hline $\mathbf{2 0 1 3}$ & 927 & $20,08 \%$ \\
\hline $\mathbf{2 0 1 4}$ & 987 & $6,47 \%$ \\
\hline $\mathbf{2 0 1 5}$ (Proyección) & 829 & $-16,02 \%$ \\
\hline
\end{tabular}

\section{DISCUSIÓN}

La planeación para optimizar los indicadores de rentabilidad (TIR promedio e IR corregido) partió de un escenario donde la situación de inversión era aceptable por la condición de que TMAR e IR eran igua les con una anualidad mínima requerida para cumplir esta condición. Esta anualidad da la posibilidad al empresario de suponer el escenario más bajo de entrada de flujo de efectivo bruto, este escenario se agrava con los montos impositivos hasta la construcción de los verdaderos flujos de efectivo, pero se verifica que existe rentabilidad a partir del análisis de la depreciación, lo cual otorga una holgura a la decisión de poder invertir o no en el activo fijo. Esta holgura es posible potenciarla a partir de la depreciación áurica, según se señala en los gráficos de TIR promedio e IR corregido promedio.

De forma general, la tasa interna de retorno promedio es mayor cuando se utiliza la depreciación áurica, puesto que la curva de regresión lineal muestra un mejor coeficiente en la variable TMAR planificada (TMARp) para este método que la depreciación acelerada y línea recta (figura 1). Un análisis de puntos para esta TIR promedio muestra que en realidad existe un nodo de puntos cercanos en los primeros valores de TMAR planificada que llevan a considerar, por ahora, que el mode lo de depreciación áurica no siempre es más factible. Sin embargo, el análisis de puntos (Tabla 6) muestra que cuando el método de línea recta es mejor que los otros dos en cuanto a mayor valor de TIR, el valor de la TMAR es más alto y no sería rentable la inversión; aún, cuando el mejor es el método de depreciación acelerada. Es del 11\% al 16\% cuando el método de depreciación acelerada provoca que sea aceptable invertir (TMAR < TIR); no obstante, la diferencia más aceptable es de apenas $0,79 \%$. Es a partir de ahí que el método de depreciación áurica es mejor para mayores valores de TIR promedio, incluso para porcentajes más altos, siendo por ejemplo $1,75 \%$ más alto en un caso de TMAR planificada para $20 \%$.

De igual forma, el índice de rentabilidad promedio corregido (IR promedio corregido) muestra un mejor desempeño por cuanto presenta un mayor valor de coeficiente para IR planificado sin impuestos, sin ser mayor que este obviamente por la razón de la aplicación impositiva. Hasta el $15 \%$ de TMAR planificada (o IR planificado) el mejor resultado, independientemente de los tres métodos, es realmente el menos inadecuado, esto porque el mejor IR corregido es negativo y de ningún modo es aceptable la inversión. A partir del valor entero de $16 \%$ de IR planificado, la rentabilidad corregida es positiva, lo que indica la existencia de recuperación de la inversión y alguna ganancia; esto se vuelve evidente a medida que se aumenta este IR planificado y se potencia 
con el uso de la depreciación áurica, pues a partir de este porcentaje es donde este método optimiza esta rentabilidad.

Se evaluó el método de depreciación áurica como aplicable a la realidad de la rentabilidad empresarial y la recaudación tributaria. Según el Instituto Nicaragüense de Investigaciones y Estudios Tributarios (INIET, 2015), los cambios en el Impuesto sobre la Renta, Impuesto al Valor Agregado, al comercio exterior, princ ipa lmente el Impuesto Selectivo al Consumo, y los aranceles de protección temporal, aunado al creciente e incontrolado proceso de otorgamiento de exoneraciones tributarias, caracterizan una tendencia destinada en lo esencial a preservar intereses de élites económicas y políticas en detrimento de sectores medios y de una población altamente vulnerable. Lo cual se evidencia en la Tabla 8, pues el crecimiento de formalización de empresas en el 2014 en la ciudad de Managua apenas es del 6,47\% respecto al año anterior y mediante esta tendencia se prevé en el 2015 un decremento, para tales efectos son muchos los factores que están relacionados a este bajo crecimiento, pero uno de los de mayor repercusión es el impuesto, debido a que no existen políticas fiscales que funcionen como incentivos a todas aquellas personas que dispongan de la creación de una empresa, por ende es necesario realizar algunos cambios al sistema fiscal.

Uno de los aspectos que pueden dinamizar la economía nicaragüense es el capital humano, es decir la creación de nuevas empresas, que por lo general la primera etapa (primeros 5 años) pueden llegar a fracasar debido a que en este punto se debe realizar una inversión anidado a los diferentes gastos para poder operar, por lo tanto no logran capitalizar lo que conlleva a la opción aparentemente viable de cerrar operaciones. Los activos adquiridos en este periodo de tiempo se deprecian y este es uno de los costos deducibles a la renta gravable, lo cual tiene un gran impacto en el pago de impuestos. Mediante el consolidado de resultados a manera de comparación, el sistema actual (método de línea recta) no provee ningún incentivo a estas empresas que están en crecimiento, el método de depreciación acelerada contempla ciertos beneficios, pero no es aplicable a todo tipo de sector y finalmente la depreciación áurica está por encima de estos dos métodos y puede funcionar como incentivo fiscal, debido que los impuestos que se pagan en los primeros años después de adquirido el activo en la mayoría de los casos es menor como se puede observar en el ejemplo de la Tabla 5, permitiendo a la empresa que está en proceso de crecimiento capitalizar en este primer año lo que le da mayor estabilidad, capacidad de seguir operando y la opción de reinvertir su dinero en todo aquello que le genere renta.

Bajo el precepto de que la depreciación áurica funciona como incentivo fiscal para aquellas empresas que están en proceso de crecimiento, se puede contrarrestar la tendencia negativa del índice de creación de nuevas empresas. Ante tal efecto, puede existir una contradicción al verse afectada la recaudación tributaria; sin embargo al aumentarse el índice de creación de empresas, también se incrementará la recaudación tributaria ya que habrá mayor cantidad de empresas y esto incide en la cantidad recaudada, explicado por la sinergia, lo que consiste en que la suma de las partes es mucho mayor que la porción individual. Además de ello, el modelo de depreciación áurica deja una premisa: una vez que la empresa logró capitalizarse, el pago de impuestos será mayor a como se evidencia en la Tabla 5, pero esto no afecta a la empresa dado que en este punto se encuentra en condiciones óptimas para poder afrontar esta situación.

Nexo Revista Científica Vol. 28, No. 02, pp. 67-82/Diciembre 2015 


\section{CONCLUSIONES}

Se realizó la formulación del modelo de depreciación áurico bajo la consideración de la sucesión de Fibonacci con base a los años de vida útil de los activos fijos contemplados en la LCT y se comprobó que ofrecía un mejor valor de tasa interna de retorno promedio (TIR promedio) que los métodos aplicados de depreciación por línea recta y la depreciación acelerada, a pesar de la consideración de planificar una TMAR más alta por la afectación de los montos impositivos. Esta TMAR es del 16\% aproximadamente.

Respecto al índice de rentabilidad de las inversiones, se consideró que los métodos de depreciación por línea recta y la depreciación acelerada son los menos adecuados, porque aunque su valor de IR corregido era mayor que la depreciación áurica, ésta los supera cuando se tiene una TMAR planificada también superior al $16 \%$.

El modelo de depreciación áurica incentiva las inversiones en activos fijos para la creación y formalización de nuevas empresas, atacando un probable decremento de éstas en los últimos años, y capitalizándose durante los primeros a fin de cumplir más adelante con sus obligaciones tributarias. Esto beneficia al fisco a largo plazo, pues al lograrse la perpetuidad de la empresa más allá de su etapa de maduración, se garantiza el cobro de impuestos con menores escudos fiscales, es decir, con mayor monto de recaudación.

\section{RECOMENDACIONES}

Se aconseja a las nuevas empresas el uso de la depreciación áurica para los cálculos de la depreciación de los activos fijos a fin de provocar una corrección sobre el valor contable de éstos, pues la obsolescencia y el deterioro son factores que, ponderados en el tiempo, ocasionan una menor capacidad productiva que puede verse reflejada en este modelo de depreciación.

Una recomendación válida a la administración tributaria nacional es el incentivo para la creación y formalización de nuevas empresas a través de medidas fiscales, una de las cuales podría ser la promoción de esta depreciación áurica, pues permite la capitalización de la empresa, su supervivencia y garantiza, a largo plazo, que se puedan cumplir las obligaciones tributarias con menos escudos fiscales.

Una orientación clara sobre cómo utilizar el modelo sería plantear una tasa atractiva de rendimiento superior al 16\%, porque vencido este escenario difícil, con la depreciación áurica es posible obtener índices de rentabilidad muy buenos y tasas internas de retorno adecuadas. Recuérdese, además, que se parte de un supuesto de anualidad mínima requerida, sin embargo, los flujos se vieron optimizados por este modelo.

\section{AGRADECIMIENTO}

De forma especial, se agradece a la Oficina de Información Pública del Ministerio de Fomento Industria y Comercio por ser el canal que facilitó la información sobre el registro de empresas en la Ventanilla Única de Inversiones de Managua.

\section{REFERENCIAS:}

Baca, G. (2007). Fundamentos de Ingeniería Económica (4a. ed.). México D.F.: McGraw-Hill.

Garza, J. (2014). Análisis multicriterio de puntos de inflexión de precio en el mercado de divisas. San Nicolás de los Garza, Nuevo León: Universidad Autónoma de Nuevo León. 
Gomero, N. (2014). Análisis económico de los impuestos: Impacto en la rentabilidad de las inversiones. Quipukamayoc, 79-87.

Instituto Nicaragüense de Investigaciones y Estudios Tributarios. (2015). Balance de la Ley de Concertación Tributaria. Managua, Nicaragua: INIET.

La Gaceta. Diario Oficial. (2013). Decreto 01-2013. Reglamento de la Ley No. 822, Ley de Concertación Tributaria. Managua: La Gaceta.

Rosillo, J. (2005). La inflación: ¿Elemento inocuo en las decisiones de inversión? Forum Empresarial, 20-46.

Ruiz, J. (1992). Capital y depreciación: Una aproximación endógena. Valencia, España: Universitat de Valencia.

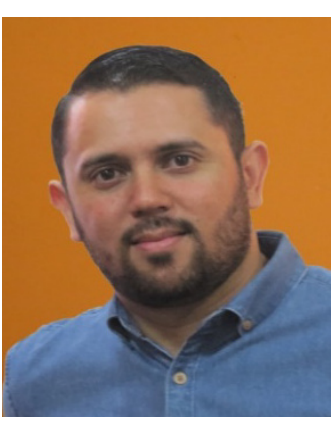

Freddy M. Silva Sobalvarro, es graduado de ingeniería industrial por la Universidad Nacional de Ingeniería (UNI) y recibió el título de maestro en finanzas por la Universidad Nacional Autónoma de Nicaragua (UNAN-Managua). Ejerce tareas de docencia e investigación a nivel de pregrado y posgrado y actualmente es doctorante de ciencias económicas por la facultad de ciencias económicas y sociales de la Universidad del Zulia (LUZ), Maracaibo, Venezuela.

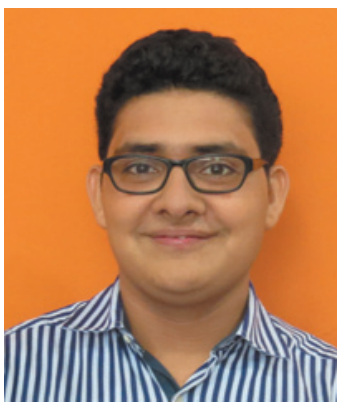

Gerald A. Marenco es estudiante de Ingeniería Industrial. Miembro de Excelencia Académica de la Facultad de Ciencia, Tecnología y Ambiente de la Universidad Centroamericana (UCA). Ganador del concurso de Nomenclatura Química y de Jóvenes Investigadores. Desarrolla trabajos de investigación concernientes a finanzas, estudios de tiempo, movimientos y teoría de colas; y participa en los talleres de Emprendimiento de la UCA.

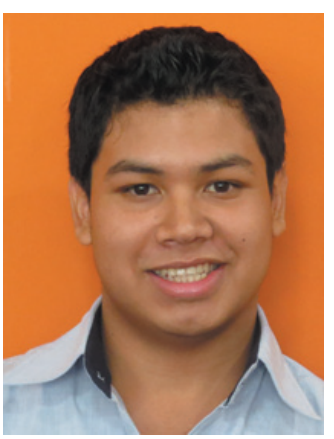

Carlos E. Zelaya es estudiante de Ingeniería Industrial. Miembro de Excelencia Académica de la Facultad de Ciencia, Tecnología y Ambiente de la Universidad Centroamericana (UCA). Ganador de Jóvenes Investigadores. Desarrolla trabajos de investigación concernientes a finanzas, estudios de tiempo, movimientos y teoría de colas; y participa en los talleres de Emprendimiento de la UCA. 